\title{
Twist-induced Near-field Thermal Switch Using Nonreciprocal Surface Magnon-Polaritons
}

\author{
Jiebin Peng, ${ }^{\dagger}$ Gaomin Tang, ${ }^{*, \dagger}$ Luqin Wang, ${ }^{\dagger}$ Rair Macêdo, ${ }^{\uparrow}$ Hong Chen, ${ }^{\dagger}$ \\ and Jie Ren*,†
}

$\dagger$ Center for Phononics and Thermal Energy Science, China-EU Joint Center for Nanophononics, Shanghai Key Laboratory of Special Artificial Microstructure Materials and Technology, School of Physics Science and Engineering, Tongji University, 200092 Shanghai, China

$\ddagger$ Department of Physics, University of Basel, Klingelbergstrasse 82, CH-4056 Basel, Switzerland

ๆJames Watt School of Engineering, Electronics \& Nanoscale Engineering Division, University of Glasgow, Glasgow G128QQ, United Kingdom

E-mail: gaomin.tang@unibas.ch; xonics@tongji.edu.cn

\begin{abstract}
We explore that two ferromagnetic insulator slabs host a strong twist-induced near-field radiative heat transfer in the presence of twisted magnetic fields. Using the formalism of fluctuational electrodynamics, we find the existence of large twist-induced thermal switch ratio in large damping condition and nonmonotonic twist manipulation for heat transfer in small damping condition, associated with the different twist-induced effects of nonreciprocal elliptic surface magnon-polaritons, hyperbolic surface magnon-polaritons, and twist-non-resonant surface magnon-polaritons. Moreover, the near-field radiative heat transfer can be significantly enhanced by the twist-non-resonant surface magnon-polaritons in the ultra-small damping condition. Such twist-induced effect is applicable for other kinds of anisotropic slabs with timereversal symmetry breaking. Our findings provide a way to twisted and magnetic control in nanoscale thermal management and improve it with twistronics concepts.
\end{abstract}


A key component for manipulating radiative heat flow at the nanoscale is near-field radiative heat transfer, which can exceed Planck's blackbody radiation limit ${ }^{1}$ by orders of magnitude due to the presence of evanescent modes. ${ }^{2-12}$ Two types of surface modes have been commonly studied in near-field heat transfer; one is surface plasmon-polaritons ${ }^{13-23}$ and the other is surface phononpolaritons. ${ }^{24-29}$ In addition, surface magnon-polaritons (SMPs), hybrid collective excitations due to the coupling between magnons and electromagnetic fields, ${ }^{30-32}$ also has functional associations to thermal management in nanotechnologies. For instance, in magnetic recording devices, a magnetic read/write head touches above the disk surface with nanometers separation. At such a short distance, SMPs should play a significant role in the near-field thermal manipulation of magnetic recording devices. Moreover, due to the high gyrotropic optical effect, ${ }^{33}$ SMPs in uniaxial ferromagnetic insulator (FMI) are nonreciprocal. Such nonreciprocal behavior can break Kirchhoff's $\mathrm{law}^{34}$ and paves the way for the exploitation of radiative thermal transfer at nanoscale.

Recently, twistronics becomes an emerging research topic since the electronic state can be manipulated through the "twist angle" between two layers, leading to flat-band superconductivity, ${ }^{35,36}$ moiré excitons, ${ }^{37}$ stacking-dependent interlayer magnetism ${ }^{38}$ and other exotic electronic properties. Similar twist-induced concepts have been demonstrated in photonics, such as moiré photonics crystal, ${ }^{39}$ moiré hyperbolic metasurfaces ${ }^{40}$ and photonic magic angles. ${ }^{41,42}$ Motivated by these exotic discoveries, several works have shown the development of tunable radiative heat flow between two-dimensional materials and biaxial crystals ${ }^{43-47}$ through twist. With the analogous principle, we explore the effects of radiative thermal twistronics between the uniaxial FMIs with external magnetic fields, where the twist and nonreciprocal phenomena can both arise in the domain of thermal management.

In this Letter, we consider to manipulate near-field radiative heat transfer through the twist between two uniaxial FMIs. Nonreciprocal SMPs emerge at the interface between vacuum and gyrotropic FMIs with asymmetric permeability tensor. Based on the nonreciprocity, we demonstrate a large twist-induced near-field thermal switch effect with a moderate external magnetic field. Under ultra-small damping condition, we show an unusual twist-induced near-field thermal transfer 
enhancement due to the presence of twist-non-resonant SMPs.

(a)

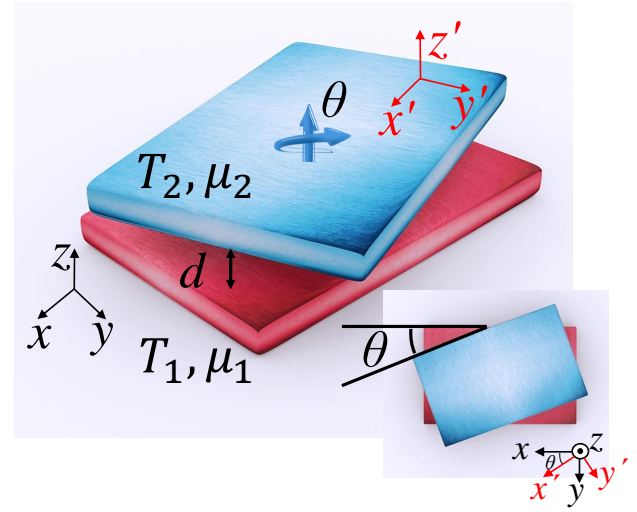

(c)

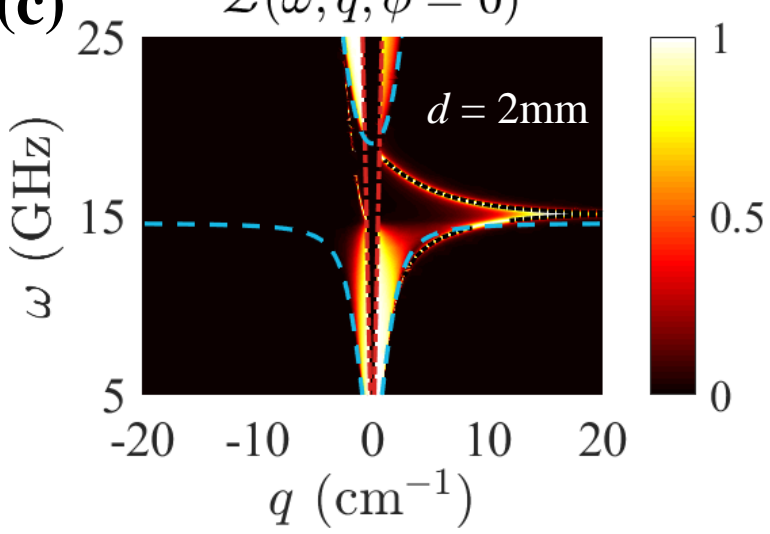

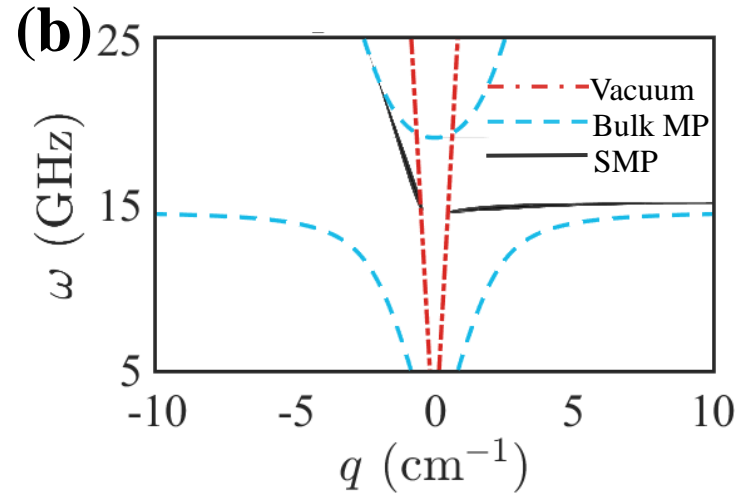

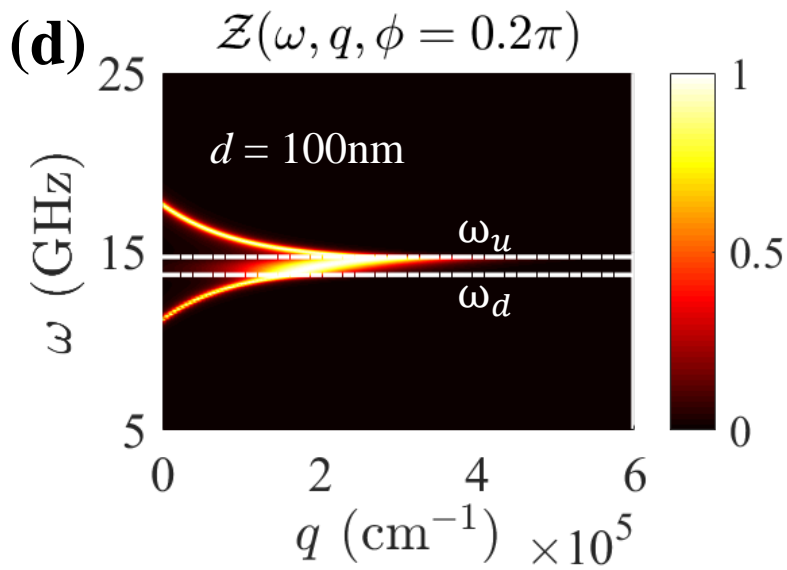

Figure 1: (a) A schematic setup for radiative heat transfer between two FMIs with vacuum separation $d$. The bottom and top slabs have the temperature $T_{1}$ and $T_{2}$, respectively. The $y\left(y^{\prime}\right)$ axis is along the direction of the satuation magnetisation in the bottom (top) FMI. The magnetic fields in each slab is applied along the direction of the corresponding satuation magnetization. The twist angle $\theta$ is defined by the anticlockwise rotation of $x^{\prime} y^{\prime} z^{\prime}$ coordinate system with respect to $x y z$ coordinate system. (b) Dispersion relation of nonreciprocal SMP with a single vacuum-FMI interface. (c) Energy transmission coefficient $\mathcal{Z}(\omega, q, \phi=0)$ with gap distance $d=2 \mathrm{~mm}$. The cyan dashed line and the red dash-dotted line are the same as in (b). The black dotted line shows the nonreciprocal symmetric and asymmetric modes of SMPs. (d) Energy transmission coefficient $\mathcal{Z}(\omega, q, \phi=0.2 \pi)$ with gap distance $d=100 \mathrm{~nm}$. The damping constant $\alpha$ is $0.01 \mathrm{in}$ (c) and (d).

Radiative heat transfer-- We consider near-field radiative heat transfer between two FMIs with temperatures $T_{1(2)}=T \pm \Delta T / 2$ and twist angle $\theta$ [See Fig. 1(a)]. A Cartesian coordinate system $x y z\left(x^{\prime} y^{\prime} z^{\prime}\right)$ is defined at the bottom (top) slab and the $y\left(y^{\prime}\right)$ axis is along the direction of the applied magnetic field and saturation magnetisation. The twist angle $\theta$ is defined as the angle between the $y^{\prime}$ and $y$ axis. We define the heat transfer coefficient $\kappa$ as $\kappa=\lim _{\Delta T \rightarrow 0} J / \Delta T$ with $J$ 
the heat flux. From fluctuational electrodynamics, ${ }^{3,4}$ the heat transfer coefficient can be expressed as

$$
\kappa(T, \theta)=\int_{0}^{\infty} \frac{d \omega}{2 \pi} \hbar \omega \frac{\partial N}{\partial T} \int_{0}^{\infty} \frac{d q}{2 \pi} q \int_{0}^{2 \pi} \frac{d \phi}{2 \pi} Z(\omega, q, \phi),
$$

where $q$ is the in-plane wave vector and $\phi$ the in-plane azimuthal angle. In the above expression, $\partial N / \partial T$ is the derivative of the Bose distribution function with respect to the temperature. We consider the relative heat transfer coefficient scaled by the black-body limit $\kappa_{b}=4 \sigma_{b} T^{3}$ with $\sigma_{b}=\pi^{2} k_{B}^{4} /\left(60 \hbar^{3} c^{2}\right)$. The energy transmission coefficient $\mathcal{Z}(\omega, q, \phi)$ with twist angle $\theta$ reads

$$
\mathcal{Z}= \begin{cases}\operatorname{Tr}\left[\left(\mathbf{I}-\mathbf{R}_{2}^{\dagger} \mathbf{R}_{2}\right) \mathbf{D}\left(\mathbf{I}-\mathbf{R}_{1} \mathbf{R}_{1}^{\dagger}\right) \mathbf{D}^{\dagger}\right], & q<\omega / c \\ \operatorname{Tr}\left[\left(\mathbf{R}_{2}^{\dagger}-\mathbf{R}_{2}\right) \mathbf{D}\left(\mathbf{R}_{1}-\mathbf{R}_{1}^{\dagger}\right) \mathbf{D}^{\dagger}\right] e^{-2\left|\beta_{0}\right| d}, & q>\omega / c\end{cases}
$$

where $\beta_{0}=\sqrt{(\omega / c)^{2}-q^{2}}$ is the out-of-plane wave vector in vacuum and $\mathbf{I}$ the identity matrix. The Fabry-Perot-like denominator matrix is written as $\mathbf{D}=\left(\mathbf{I}-\mathbf{R}_{1} \mathbf{R}_{2} e^{2 i \beta_{0} d}\right)^{-1}$. In our setup, the reflection coefficient matrix $\mathbf{R}_{a}$ with $a=1,2$ is written as

$$
\mathbf{R}_{a}=\left[\begin{array}{cc}
r_{s s}^{a} & r_{s p}^{a} \\
r_{p s}^{a} & r_{p p}^{a}
\end{array}\right]
$$

where superscripts $s$ and $p$ denote the polarization states. The reflection coefficients can be calculated by the transfer matrix methods ${ }^{48}$ and the details are given in the Supplemental Material. ${ }^{49}$ For later convenience, we also define the integrated energy transmission coefficient, i.e. $\mathcal{Z}(\omega, \phi)$, which is the energy transmission coefficient after an integration over the wave vector $q$.

By applying a magnetic field along the $y$-direction in the bottom FMI, the permeability tensor has the form ${ }^{31,33}$

$$
\mu=\left[\begin{array}{ccc}
\mu_{x x} & \mu_{x y} & \mu_{x z} \\
\mu_{y z} & \mu_{y y} & \mu_{y z} \\
\mu_{z x} & \mu_{z y} & \mu_{z z}
\end{array}\right]=\left[\begin{array}{ccc}
\mu_{r} & 0 & -i \mu_{i} \\
0 & 1 & 0 \\
i \mu_{i} & 0 & \mu_{r}
\end{array}\right]
$$


The diagonal and off-diagonal terms are, respectively, expressed as $\mu_{r}=1+\frac{\omega_{m}\left(\omega_{0}+i \alpha \omega\right)}{\left(\omega_{0}+i \alpha \omega\right)^{2}-\omega^{2}}$ and $\mu_{i}=\frac{\omega_{m} \omega}{\left(\omega_{0}+i \alpha \omega\right)^{2}-\omega^{2}}$ with frequency $\omega$ and magnetic precession damping constant $\alpha$. The magnetic resonance frequencies $\omega_{0}=\mu_{0} \gamma h$ and $\omega_{m}=\mu_{0} \gamma m_{s}$ are due to the external magnetic field $h$ and the saturation magnetization $m_{s}$ with the gyromagnetic ratio $\gamma$. The relative permittivity of the FMI is assumed to be a constant. For the top FMI, the relative permeability tensor is expressed as $\mu^{\prime}=\mathcal{R}(\theta) \mu \mathcal{R}^{T}(\theta)$ with the rotation matrix $\mathcal{R}(\theta)$ along $z$ axis. During the numerical calculation, we adopt the parameters of yttrium iron garnet (YIG) with the relative permittivity $\epsilon=14.5,{ }^{50} \mathrm{gy}$ romagnetic ratio $\gamma / 2 \pi=28 \mathrm{GHz} / \mathrm{T}^{51}$ and saturation magnetization $\mu_{0} m_{s}=0.28 \mathrm{~T} .{ }^{52}$ The applied magnetic field $\mu_{0} h$ is taken as $0.4 \mathrm{~T}$. Such set of parameters results in SMPs at microwave frequency range so that we consider the radiative heat transfer at the cryogenic environment (around $4 \mathrm{~K})$.

Nonreciprocal surface magnon-polaritons.- At a single vacuum-FMI interface, there exists SMPs of which the dispersion is nonreciprocal. The implicit dispersion relation for SMPs is ${ }^{49}$

$$
\beta_{0}+\left(\mu_{r} \beta_{1}-i \mu_{i} q\right) /\left(\mu_{r}^{2}-\mu_{i}^{2}\right)=0
$$

where $\beta_{1}=\sqrt{\epsilon \mu_{e f f}(\omega / c)^{2}-q^{2}}$ is the out-of-plane wave vector inside the FMI and $\mu_{e f f}=\left(\mu_{r}^{2}-\right.$ $\left.\mu_{i}^{2}\right) / \mu_{r}$. Figure. 1(b) indicates the nonreciprocal dispersion of SMPs (gray line) outside the light cone (red dash-dotted line), together with the symmetric dispersion of bulk magnon-polations, that is, $q=\sqrt{\epsilon \mu_{e f f}} \omega / c$ (cyan dashed line). We highlight that SMPs exist at the band gap region of FMI and the high- $q$ SMPs only exist at positive wave vector region, which is useful in manipulating near-field heat transfer.

For the case of two FMIs with millimeter separation, SMPs from two interfaces can be coupled. Figure. 1(c) shows the energy transmission coefficient between two FMIs at zero azimuthal and twist angle, that is, $\mathcal{Z}(\omega, q, \phi=0 ; \theta=0)$. We can observe that there exists an asymmetric transmission coefficient both for bulk MPs (the region outside the light cone and inside the dispersion relation of MPs) and SMPs (the near-unity line inside the band gap), with respect to the in-plane 
wave vector. The two near-unity lines for SMPs are consistent with the implicit dispersion relation of SMPs as follows

$$
\begin{aligned}
& \beta_{0}+\tanh \left(\left|\beta_{0}\right| d / 2\right)\left(\beta_{1} \mu_{r}-i q \mu_{i}\right) /\left(\mu_{r}^{2}-\mu_{i}^{2}\right)=0 \\
& \beta_{0}+\operatorname{coth}\left(\left|\beta_{0}\right| d / 2\right)\left(\beta_{1} \mu_{r}-i q \mu_{i}\right) /\left(\mu_{r}^{2}-\mu_{i}^{2}\right)=0 .
\end{aligned}
$$

In the absence of the contributions from $\mu_{i}$, Eqs. (6) and (7) can be reduced to dispersion relations similar to those of surface phonon-polaritons.

In addition, the optical properties of FMI are anisotropic in the $x$ - $z$ plane when there is nonzero azimuthal angle. To qualitatively analyze the anisotropic effects, we show the energy transmission coefficient with a nonzero azimuthal angle in Fig. 1(d), where the near-unity lines between frequency $\omega_{u}$ and $\omega_{d}$ expand as a near-unity spot. Here, $\omega_{u}$ and $\omega_{d}$ are the $\mu$-near-zero frequencies with azimuthal angles $\phi=0$ and $\phi=0.2 \pi$, respectively, and are determined by $\mu_{r}\left(\omega_{u / d}\right) \cos ^{2} \phi+\sin ^{2} \phi=0$. In the region between $\omega_{u}$ and $\omega_{d}$, the diagonal terms of permeability tensor in $x-z$ plane have the opposite sign, that is, $\mu_{x x}>0, \mu_{y y}>0$ and $\mu_{z z}<0 .{ }^{49}$ It is similar to type-I hyperbolic metamaterial ${ }^{45,53}$ without considering the off-diagonal term in the permeability tensor. Comparing with that of $\phi=0$ condition, i.e., $\mu_{x x}<0, \mu_{y y}>0$ and $\mu_{z z}<0$, the twistinduced hyperbolic SMPs emerge at $x$ - $z$ plane when $\phi=0.2 \pi$. Fig. 1(d) proves the existence of such hyperbolic SMPs and also shows that it can provide more channels for radiative heat transfer. So this azimuthal-angle dependent hyperbolic mode can contribute to a enhancement of radiative heat transfer. The coexistence of nonreciprocal and anisotropic effects in FMI is helpful for twisted and magnetic thermal management.

Twist-induced Near-field Thermal Switch.- To study the twist-induced thermal switch mediated by the nonreciprocal SMPs, the thermal switch ratio $R_{\kappa}(\theta)$ is defined as

$$
R_{\kappa}(\theta)=\kappa(\theta) / \kappa_{\min }
$$

where $\kappa_{\min }$ is the minimal heat transfer coefficient by changing the twist angle $\theta$. 

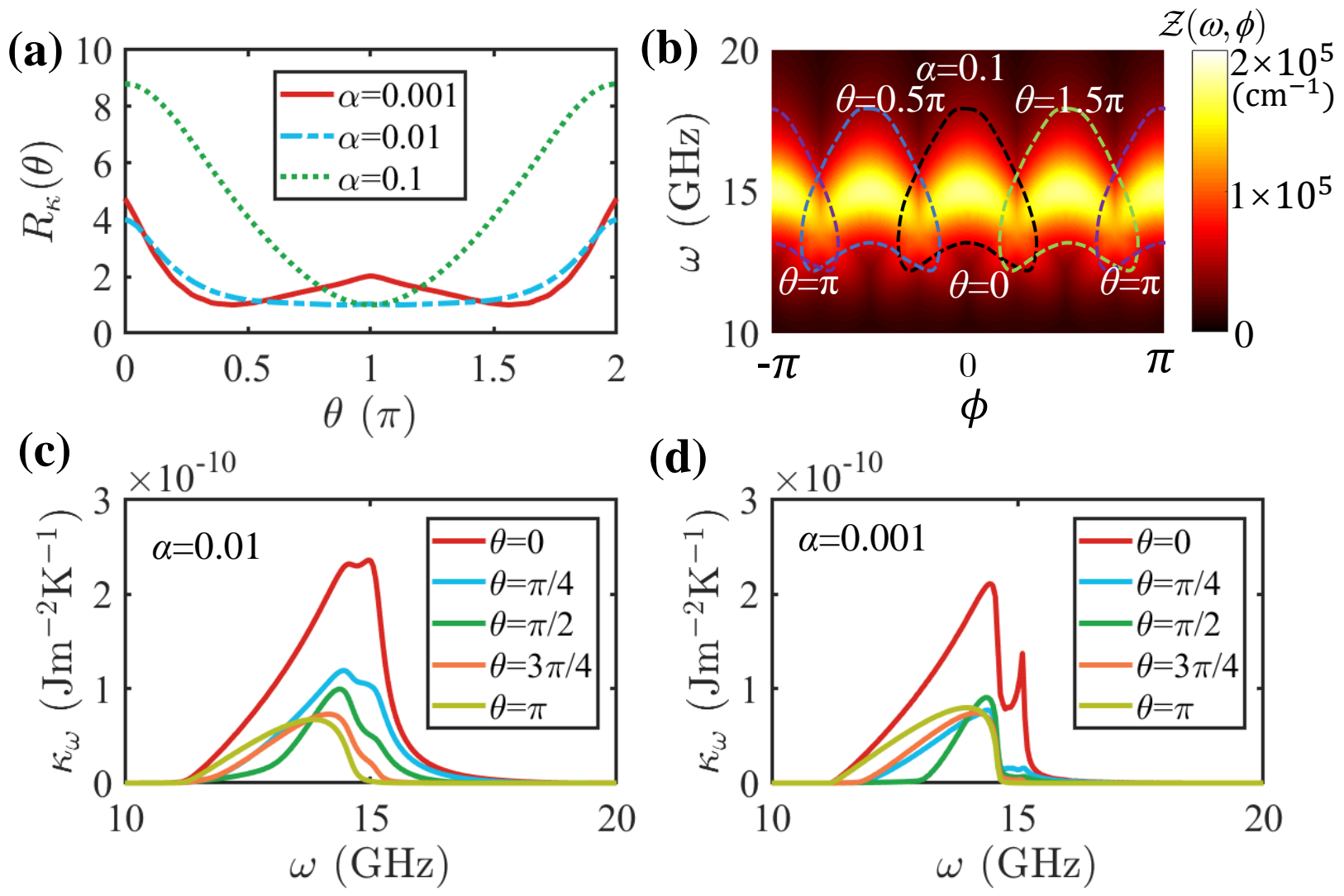

Figure 2: (a) Twist-induced near-field thermal switch ratio as a function of twist angle with different damping constants $\alpha$. (b) The contour for integrated energy transfer coefficient in $\omega-\phi$ space at single vacuum-FMI interface with different twist angles. (c)-(d) The spectral function of heat transfer coefficient with different damping constants and twist angles. 
Fig. 2(a) shows the switch ratio with different damping constants $\alpha$. It can be seen that the switch ratio is maximal at the parallel configuration $(\theta=0)$. At large damping conditions, the green-dotted line in Fig. 2(a) indicates that the switch ratio reaches about 9. The physical mechanism of such a large switch ratio can be related to the match or mismatch of the integrated energy transmission coefficient in the $\omega-\phi$ space. As shown in Fig. 2(b), the overlap region of the integrated energy transmission coefficient reaches maximal value in parallel configuration. With increasing or decreasing the twist angle $\theta$, the central region of the integrated energy transmission coefficient at the twisted FMI will shift left or right in $\omega$ - $\phi$ space and the overlap between two FMIs reaches the minimum value in anti-parallel configuration. These twist-induced mismatch effects result in a large thermal switch ratio.

Under a small damping, the switch ratio is nonmonotonic with respect to the twist angle, as indicated by the red solid line in Fig. 2(a). Such angle-dependent behavior is similar to the thermal magnetoresistance between two magneto-optical plasmonic particles at a large applied magnetic field. ${ }^{54}$ To explore this different angle dependence at small damping condition, we show the spectral function $\kappa_{\omega}$ by varying the twist angle in Figs. 2(c) and 2(d). The twist angle strongly modulates the height and the width of the spectral function peaks at $0<\theta<\pi / 2$. However, when $\pi / 2<\theta<\pi$, the high-frequency peak in spectral function almost disappear and the width of the low-frequency peak becomes broader with $\theta$ increasing. Such results qualitatively indicate that there are several nonreciprocal SMPs taking part in the heat transfer with different angle dependence. The isofrequency contour for energy transmission coefficient at $q_{x}-q_{y}$ space in Fig. 3(a) numerically verify that statement and we show three kinds of SMPs: elliptic SMPs, hyperbolic SMPs, ${ }^{53}$ and twist-non-resonant SMPs. The different twist-induced tunneling and competition between those modes lead to above nonmonotonic twist manipulation for heat transfer.

Figure 3(a) shows the different twist-induced energy transmission coefficient of the above mentioned SMPs in $q_{x}-q_{y}$ space. Due to the nonreciprocal properties of SMPs, the tunneling of three kinds of SMPs only occur at a positive $q_{x}$ region, except in antiparallel configuration. On the one hand, the vertical slice contours in Fig. 3(a) indicate that there is a transition between hy- 

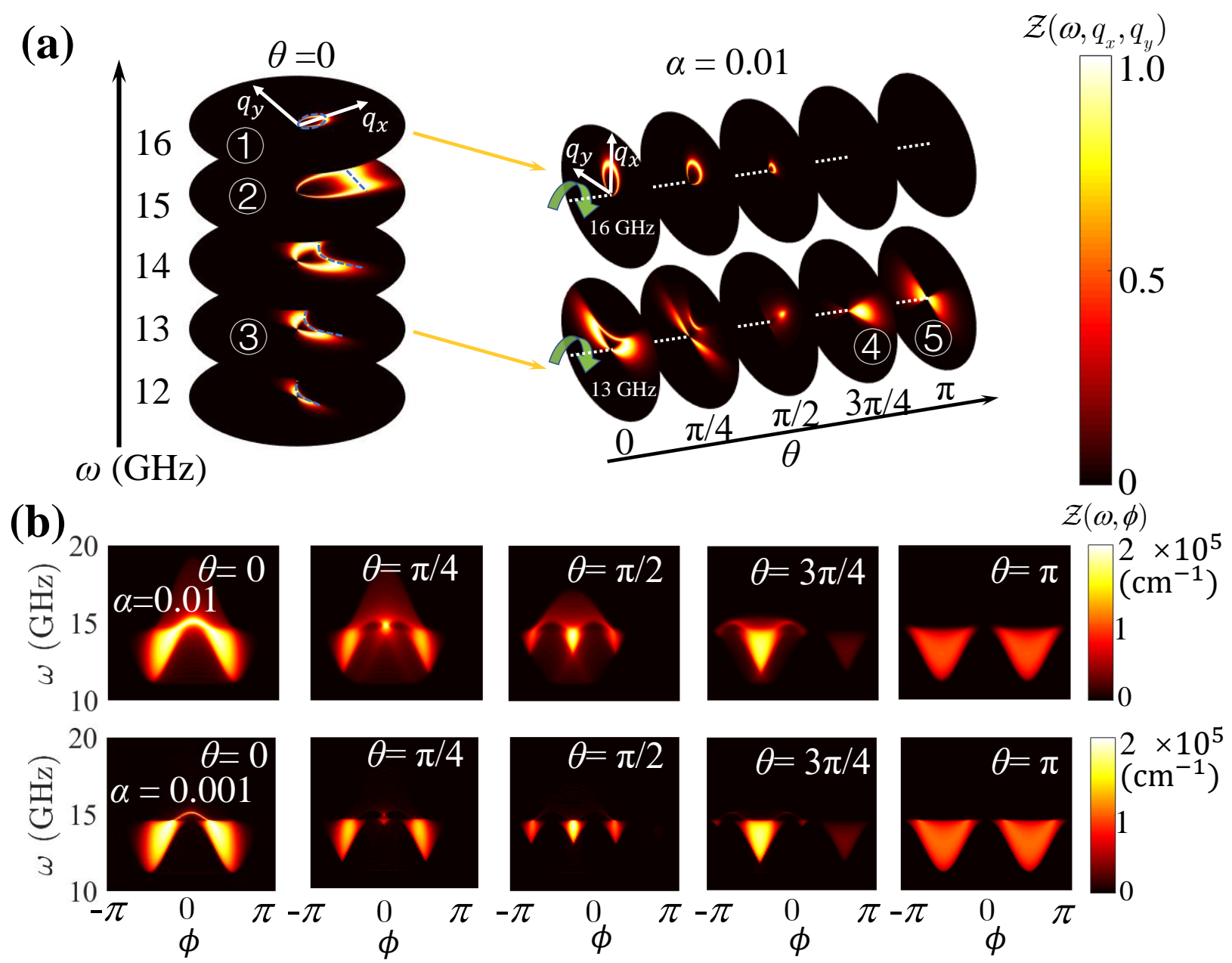

Figure 3: (a) Twist-induced energy transmission coefficient with different frequency in $q_{x}-q_{y}$ space. Left-vertical slice figures are the energy transmission coefficient with zero twist angle with frequency increasing. Right-transverse slice figures are the energy transmission coefficient at fixed frequency with the twist angle increasing. (b) Integrated energy transmission coefficient in $\omega-\phi$ space with different twist angles and damping constants. 
perbolic SMPs and elliptic SMPs with an increase of frequency. We highlight that the isofrequency contours of the energy transmission coefficient can be almost flat at $\omega \approx 15 \mathrm{GHz}$ and result in a sharp peak in the spectral function (Figs. 2(c) and 2(d)). In that scenario, such flattening transition behavior allows the SMPs bands of each individual FMI hybridize and strongly coupled to each other with large wavenumbers and involves a dramatic increase of the local density of states for near-field radiative heat transfer. On the other hand, Fig. 3(a) also indicates that the elliptic SMPs and hyperbolic SMPs propagate at the open-angle $\left(-\phi_{m}<\phi<\phi_{m}\right)$, where $\phi_{m}=\arctan \sqrt{1 /\left[\mu_{i}(\omega)-\mu_{r}(\omega)\right]}$. But the twist-non-resonant SMPs emerge when $\omega<15 \mathrm{GHz}$ and is not bounded by the open-angle $\phi_{m}$ because it originates in the twist-induced anisotropic in $x-z$ plane. The horizontal slice figures in Fig. 3(a) demonstrate the twist-induced effects of three kinds of SMPs: monotonically decreasing for elliptic SMPs and hyperbolic SMPs and nonmonotonic dependence for twist-non-resonant SMPs at $0<\theta<\pi$. The competition mechanism among three kinds of modes can be understood from the integrated energy transmission coefficient in $\omega$ - $\phi$ space with different damping constants (Fig. 3(b)). When $\alpha=0.01$, elliptic SMPs, and hyperbolic SMPs play an equal role for radiative heat transfer comparing with twist-non-resonant SMPs, which leads to an almost monotonically decreased thermal switch ratio. In the small damping condition, i.e., $\alpha=0.001$, the twist-non-resonant SMPs will play the dominant role for radiative heat transfer, which is induced by the optical gyrotropy and leads to a $\theta$ anisotropy in the radiative heat transfer.

Besides, we find an optimal damping constant for maximizing the heat transfer coefficient in Fig. 4(a): the magnitude of heat current can be enhanced almost one order in ultra-small damping condition comparing with the isotropic case and the heat flux is monotonically decreased at antiparallel configuration $(\theta=\pi)$. Based on fluctuation electrodynamics, heat flux between two semi-infinite systems is proportional to the imaginary part of the permeability and the magnitude of heat current could be reduced to zero when the damping constant approach zero or a large value. But the heat transfer coefficient between two FMIs reaches a fixed value in zero damping constant limit. We demonstrate that twist-non-resonant SMPs play the dominant role in ultra-small damp- 
(a)

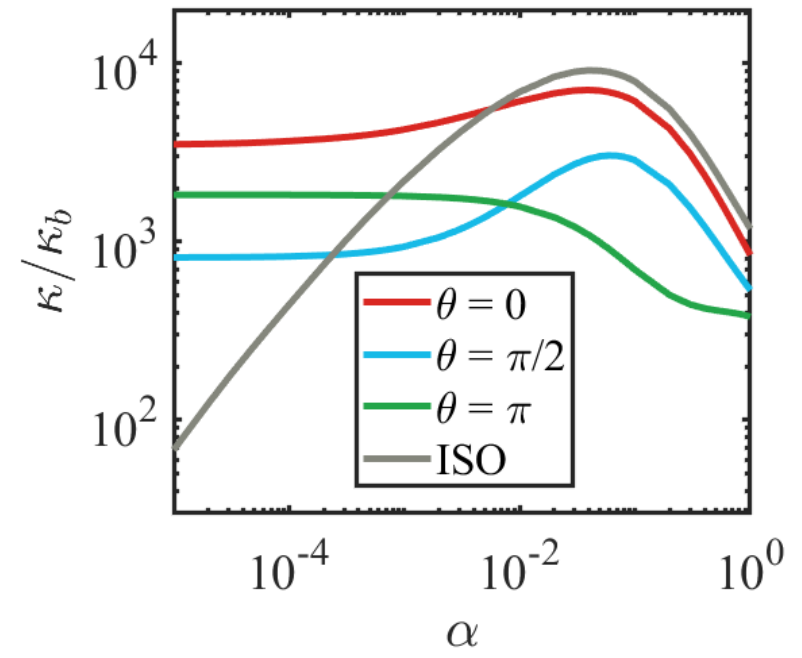

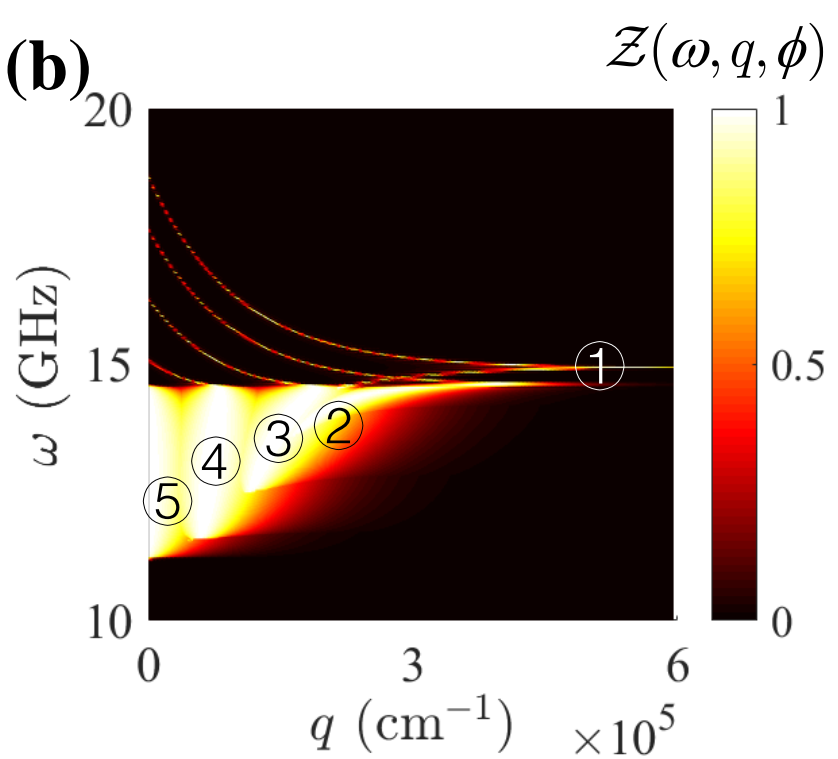

Figure 4: (a) Heat transfer coefficient as a function of damping constant $\alpha$ with different twist angle. Gray-solid line is the heat transfer coefficient between two isotropic slab i.e. $\mu_{x x}=\mu_{y y}=$ $\mu_{z z}$. (b) Energy transmission coefficient in $\omega-q$ space with different azimuthal angle $\phi$. (1)-(5)) means that the azimuthal angles $\phi$ are from $0.1 \pi$ to $0.5 \pi$ with step $0.1 \pi$, respectively. The twist angle $\theta$ is zero and the damping constant $\alpha$ is 0.001 .

ing conditions and the mechanism is slightly different from near-field radiative heat transfer in multilayer structure due to multiple surface-states coupling. ${ }^{55}$ The intrinsic relation between twistnon-resonant SMPs and the heat transfer coefficient is demonstrated at Fig. 4(b): the near-unity region in energy transmission coefficient contour with different azimuthal angle can fill in the giant area in $\omega-q$ space and the local density of states for twist-non-resonant SMPs can be boosted without the constraint of ultra-small damping condition. It also demonstrates that the local density of states for elliptic SMPs and hyperbolic SMPs (the thin-solid line in Fig. 4(b)) is not be enhanced and plays little contribution for heat transfer in a ultra-small damping condition. As a whole, the different $\alpha$ and $\theta$ dependence of elliptic SMPs, hyperbolic SMPs, and twist-non-resonant SMPs result in above twist-induced manipulation for near-field radiative heat transfer.

To conclude, we have studied twist-induced near-field radiative heat transfer between two FMIs through nonreciprocal SMPs. We find a large and nonmonotonic twist-induced near-field thermal switch ratio. In addition, the near-field radiative heat transfer can be enhanced by the contribu- 
tions from the twist-non-resonant SMPs under ultra-small damping condition. Our results provide insights for active near-field heat transfer control by engineered twists.

\section{Acknowledgement}

J.-P., L.-W., H.-C., and J.-R. are supported by the National Key Research Program of China (Grant No. 2016YFA0301101), National Natural Science Foundation of China (No. 11935010, No. 11775159 and No. 61621001), the Shanghai Science and Technology Committee (Grants No. 18ZR1442800 and No. 18JC1410900), and the Opening Project of Shanghai Key Laboratory of Special Artificial Microstructure Materials and Technology. G.-T. thanks the financial support from the Swiss National Science Foundation (SNSF) and the NCCR Quantum Science and Technology. Rair Macedo acknowledges support from the Leverhulme Trust and the University of Glasgow through LKAS funds.

\section{Supporting Information Available}

In the Supplemental Material, we derive the dispersion relation and the reflection coefficients of the surface magnon polariton.

\section{Dispersion relation of surface magnon polariton}

To find the dispersion relation of surface magnon polaritons (SMP) at a single vacuum-FMI interface, we employ Maxwell equations,

$$
\begin{aligned}
& \nabla \times \mathbf{E}=-\partial_{t} \mathbf{B}, \\
& \nabla \times \mathbf{H}=\partial_{t} \mathbf{D},
\end{aligned}
$$

with $\mathbf{B}=\mu_{0} \mu \mathbf{H}$ and $\mathbf{D}=\epsilon_{0} \epsilon \mathbf{E}$. The SMP is transverse electric (TE or $s$ ) polarized and the transverse magnetic (TM or $p$-polarized) mode does not exist for the case where only single FMI 
slab is considered. By applying an in-plane magnetic field along the $y$-direction, the electric fields in vacuum $\mathbf{E}_{0}$ and in $\mathrm{FMI} \mathbf{E}_{1}$ propagate along the $x$-direction and decay along the $z$-direction with the expressions

$$
\begin{array}{ll}
\mathbf{E}_{0}(x, z, t)=\hat{y} E e^{i q x-i \beta_{0} z} e^{-i \omega t}, & \operatorname{Im}\left(\beta_{0}\right)<0, \\
\mathbf{E}_{1}(x, z, t)=\hat{y} E e^{i q x+i \beta_{1} z} e^{-i \omega t}, & \operatorname{Im}\left(\beta_{1}\right)<0,
\end{array}
$$

where $q$ is the in-plane wave vector along the $x$-direction. The out-of-plane wave vector in vacuum and FMI are denoted as $\beta_{0}$ and $\beta_{1}$, respectively. The corresponding magnetic fields are expressed as

$$
\mathbf{B}_{0 / 1}(x, z, t)=\frac{i}{\omega}\left(\hat{x} \partial_{z}-\hat{z} \partial_{x}\right)\left(\mathbf{E}_{0 / 1} \cdot \hat{y}\right)
$$

From $\mathbf{H}=\left(\mu_{0} \mu\right)^{-1} \mathbf{B}$, the magnetic field strengths are

$$
\begin{aligned}
\mathbf{H}_{0} & =\frac{i}{\omega \mu_{0}}\left[\hat{x} \partial_{z}-\hat{z} \partial_{x}\right]\left(\mathbf{E}_{0} \cdot \hat{y}\right), \\
\mathbf{H}_{1} & =\frac{i}{\omega \mu_{0}\left(\mu_{r}^{2}-\mu_{i}^{2}\right)}\left[\hat{x}\left(-i \mu_{i} \partial_{x}+\mu_{r} \partial_{z}\right)+\hat{z}\left(-\mu_{r} \partial_{x}-i \mu_{i} \partial_{z}\right)\right]\left(\mathbf{E}_{1} \cdot \hat{y}\right) .
\end{aligned}
$$

Using Eq. (10) in both vacuum and FMI, one has

$$
\begin{aligned}
& \beta_{0}^{2}+q^{2}=k_{0}^{2}, \\
& \beta_{1}^{2}+q^{2}=\epsilon \mu_{\mathrm{eff}} k_{0}^{2},
\end{aligned}
$$

with $k_{0}=\omega / c$ and $\mu_{\mathrm{eff}}=\left(\mu_{r}^{2}-\mu_{i}^{2}\right) / \mu_{r}$. Using the interface conditions for the magnetic field strengths, $\mathbf{H}_{0} \cdot \hat{x}=\mathbf{H}_{1} \cdot \hat{x}$, the implicit dispersion relation for the SMPs can be obtained with

$$
\beta_{0}+\left(\mu_{r} \beta_{1}-i \mu_{i} q\right) /\left(\mu_{r}^{2}-\mu_{i}^{2}\right)=0
$$

From Eqs. (16), (17) and (18), the dispersion relation of SMP can be numerically obtained. 


\section{Reflection coefficients}

In this section, we obtain the reflection coefficients by taking the anisotropic effect into account. For the case where incidence plane is at an angle $\phi$ with respect to the $x$-axis, the effective permeability tensor is

$$
\mu^{\prime}=\mathcal{R} \mu \mathcal{R}^{T}=\left[\begin{array}{lll}
\mu_{x x} & \mu_{x y} & \mu_{x z} \\
\mu_{y x} & \mu_{y y} & \mu_{y z} \\
\mu_{z x} & \mu_{z y} & \mu_{z z}
\end{array}\right],
$$

where $\mathcal{R}$ is the rotation matrix with

$$
\mathcal{R}=\left[\begin{array}{ccc}
\cos \phi & \sin \phi & 0 \\
-\sin \phi & \cos \phi & 0 \\
0 & 0 & 1
\end{array}\right]
$$

Although the SMP is $s$-polarized by considering a single FMI slab, the $p$-polarized mode exists between two FMI slabs as well due to the anisotropic permeability tensor. We focus on the interface between the lower FMI slab and vacuum. The genernal form of the electric and magnetic fields inside the FMI slab can be written as

$$
\begin{aligned}
\mathbf{E} & =\left(\mathcal{E}_{x}, \mathcal{E}_{y}, \mathcal{E}_{z}\right) e^{-i \omega t+i q x}, \\
\mathbf{H} & =\left(\mathcal{H}_{x}, \mathcal{H}_{y}, \mathcal{H}_{z}\right) e^{-i \omega t+i q x},
\end{aligned}
$$

where the superscript ' in the space variables $x^{\prime}, y^{\prime}$ and $z^{\prime}$ is dropped for simplicity. From the Maxwell equations, Eqs. (9) and (10), we can get the differential equation

$$
\frac{d}{d z}\left[\begin{array}{c}
\mathcal{E}_{x} \\
\mathcal{E}_{y} \\
\alpha \mathcal{H}_{x} \\
\alpha \mathcal{H}_{y}
\end{array}\right]=i K\left[\begin{array}{c}
\mathcal{E}_{x} \\
\mathcal{E}_{y} \\
\alpha \mathcal{H}_{x} \\
\alpha \mathcal{H}_{y}
\end{array}\right]
$$


with $\alpha=\sqrt{\mu_{0} / \epsilon_{0}}$ and

$$
K=\left[\begin{array}{cccc}
0 & q \mu_{y z} / \mu_{z z} & k_{0}\left(\mu_{y x}-\mu_{y z} \mu_{z x} / \mu_{z z}\right) & k_{0}\left(\mu_{y y}-\mu_{y z} \mu_{z y} / \mu_{z z}\right)-q^{2} /\left(k_{0} \epsilon\right) \\
0 & -q \mu_{x z} / \mu_{z z} & k_{0}\left(-\mu_{x x}+\mu_{x z} \mu_{z x} / \mu_{z z}\right) & k_{0}\left(-\mu_{x y}+\mu_{x z} \mu_{z y} / \mu_{z z}\right) \\
0 & -k_{0} \epsilon+q^{2} /\left(k_{0} \mu_{z z}\right) & -q \mu_{z x} / \mu_{z z} & -q \mu_{z y} / \mu_{z z} \\
k_{0} \epsilon & 0 & 0 & 0
\end{array}\right] .
$$

By solving this differential equation, we get

$$
\left[\mathcal{E}_{x}(z), \mathcal{E}_{y}(z), \alpha \mathcal{H}_{y}(z), \alpha \mathcal{H}_{y}(z)\right]=\sum_{m=1}^{2} c_{m}\left[u_{1, m}, u_{2, m}, u_{3, m}, u_{4, m}\right] e^{i k_{m} z}
$$

where $k_{m}$ and $u_{i, m}$ are, respectively, the eigenvalue and eigenvector of matrix $K$. Since $K$ is a four-by-four matrix, we have four eigenvalues: two of them satisfy $\operatorname{Im}\left(k_{m}\right)<0$ and the other two $\operatorname{Im}\left(k_{m}\right)>0$. We take $k_{m}$ with $\operatorname{Im}\left(k_{m}\right)<0$, of which the subscripts are denoted as $m=1,2$, to ensure that the electromagnetic fields vanish at $z \rightarrow-\infty$.

In the vacuum, the incoming electric and magnetic fields can be, respectively, writen as

$$
\begin{aligned}
\mathbf{E}_{\text {in }} & =\left[e_{\text {in }}^{s} \hat{y}+e_{\text {in }}^{p}\left(\beta_{0} \hat{x}-q \hat{z}\right) / k_{0}\right] e^{i \omega t-i q x-i \beta_{0} z}, \\
\alpha \mathbf{H}_{\text {in }} & =\left[e_{\text {in }}^{p} \hat{y}-e_{\text {in }}^{s}\left(\beta_{0} \hat{x}-q \hat{z}\right) / k_{0}\right] e^{i \omega t-i q x-i \beta_{0} z},
\end{aligned}
$$

where the superscripts $s$ and $p$ are used to denote the polarizations. The reflected fields are expressed as

$$
\begin{aligned}
\mathbf{E}_{\mathrm{re}} & =\left[e_{\mathrm{re}}^{s} \hat{y}-e_{\mathrm{re}}^{p}\left(\beta_{0} \hat{x}+q \hat{z}\right) / k_{0}\right] e^{i \omega t-i q x+i \beta_{0} z}, \\
\alpha \mathbf{H}_{\mathrm{re}} & =\left[e_{\mathrm{re}}^{p} \hat{y}+e_{\mathrm{re}}^{s}\left(\beta_{0} \hat{x}+q \hat{z}\right) / k_{0}\right] e^{i \omega t-i q x+i \beta_{0} z} .
\end{aligned}
$$

At the interface of the vacuum side with $z=0^{+}$, the in-plane electric and magnetic fields can be 
written as

$$
\begin{aligned}
\mathbf{E}_{\|} & =\left[\left(e_{\mathrm{in}}^{s}+e_{\mathrm{re}}^{s}\right) \hat{y}+\left(e_{\mathrm{in}}^{p}-e_{\mathrm{re}}^{p}\right) \beta_{0} / k_{0} \hat{x}\right] e^{i \omega t-i q x}, \\
\alpha \mathbf{H}_{\|} & =\left[\left(e_{\mathrm{in}}^{p}+e_{\mathrm{re}}^{p}\right) \hat{y}-\left(e_{\mathrm{in}}^{s}-e_{\mathrm{re}}^{s}\right) \beta_{0} / k_{0} \hat{x}\right] e^{i \omega t-i q x} .
\end{aligned}
$$

For the case of the $s$-polarized incoming field, that is, $e_{\mathrm{in}}^{p}=0$, the interface conditions give

$$
\begin{aligned}
-e_{\mathrm{re}}^{p} \beta_{0} / k_{0} & =\mathcal{E}_{x}(z=0), \\
e_{\mathrm{in}}^{s}+e_{\mathrm{re}}^{s} & =\mathcal{E}_{y}(z=0), \\
-\left(e_{\mathrm{in}}^{s}-e_{\mathrm{re}}^{s}\right) \beta_{0} / k_{0} & =\alpha \mathcal{H}_{x}(z=0), \\
e_{\mathrm{re}}^{p} & =\alpha \mathcal{H}_{y}(z=0) .
\end{aligned}
$$

From Eqs. (32) and (35), we have

$$
c_{2} / c_{1}=-\left(u_{1,1} k_{0}+u_{4,1} \beta_{0}\right) /\left(u_{1,2} k_{0}+u_{4,2} \beta_{0}\right) .
$$

The reflection coefficient $r_{s s}=e_{\mathrm{re}}^{s} / e_{\mathrm{in}}^{s}$ can be obtained from Eqs. (33) and (34) as

$$
r_{s s}=\frac{\left(u_{2,1} \beta_{0}+u_{3,1} k_{0}\right)+\left(u_{2,2} \beta_{0}+u_{3,2} k_{0}\right) c_{2} / c_{1}}{\left(u_{2,1} \beta_{0}-u_{3,1} k_{0}\right)+\left(u_{2,2} \beta_{0}-u_{3,2} k_{0}\right) c_{2} / c_{1}}
$$

From Eqs. (32) and (34), we can obtain $r_{p s}=e_{\mathrm{re}}^{p} / e_{\mathrm{in}}^{s}$ as

$$
r_{p s}=\left(1-r_{s s}\right) \frac{u_{1,1}+u_{1,2} c_{2} / c_{1}}{u_{3,1}+u_{3,2} c_{2} / c_{1}}
$$


For the case of the $p$-polarized incoming field, that is, $e_{\mathrm{in}}^{s}=0$, the interface conditions give

$$
\begin{aligned}
\left(e_{\mathrm{in}}^{p}-e_{\mathrm{re}}^{p}\right) \beta_{0} / k_{0} & =\mathcal{E}_{x}(z=0), \\
e_{\mathrm{re}}^{s} & =\mathcal{E}_{y}(z=0), \\
e_{\mathrm{re}}^{s} \beta_{0} / k_{0} & =\alpha \mathcal{H}_{x}(z=0), \\
e_{\mathrm{in}}^{p}+e_{\mathrm{re}}^{p} & =\alpha \mathcal{H}_{y}(z=0) .
\end{aligned}
$$

From Eqs. (40) and (41), we have

$$
d_{2} / d_{1} \equiv c_{2} / c_{1}=-\left(u_{2,1} \beta_{0}-u_{3,1} k_{0}\right) /\left(u_{2,2} \beta_{0}-u_{3,2} k_{0}\right)
$$

Notice $c_{2} / c_{1}$ here is different from that in Eq. (36) and we denote it as $d_{2} / d_{1}$ instead. The reflection coefficient $r_{p p}=e_{\mathrm{re}}^{p} / e_{\mathrm{in}}^{p}$ obtained from Eqs. (40) and (41) is expressed as

$$
r_{p p}=\frac{\left(u_{4,1} \beta_{0}-u_{1,1} k_{0}\right)+\left(u_{4,2} \beta_{0}-u_{1,2} k_{0}\right) d_{2} / d_{1}}{\left(u_{4,1} \beta_{0}+u_{1,1} k_{0}\right)+\left(u_{4,2} \beta_{0}+u_{1,2} k_{0}\right) d_{2} / d_{1}}
$$

From Eqs. (39) and (41), we obtain $r_{s p}=e_{\mathrm{re}}^{s} / e_{\mathrm{in}}^{p}$ as

$$
r_{s p}=\left(1-r_{p p}\right) \frac{u_{3,1}+u_{3,2} d_{2} / d_{1}}{u_{1,1}+u_{1,2} d_{2} / d_{1}}
$$

\section{Near-field radiative heat transfer}

From the fluctuating electrodynamics, the radiative heat current with vacuum gap $d$ is given by

$$
J=\int_{0}^{\infty} \frac{d \omega}{2 \pi} \hbar \omega\left(N_{1}-N_{2}\right) \int_{0}^{\infty} \frac{d q}{2 \pi} q \int_{0}^{2 \pi} \frac{d \phi}{2 \pi} \mathcal{Z}(\omega, q, \phi)
$$


where $N_{i}=1 /\left[e^{\hbar \omega /\left(k_{B} T_{i}\right)}-1\right]$ with $i=1,2$ is the Bose-Einstein distribution function. The photonic transmission coefficient $\mathcal{Z}(\omega, q, \phi)$ reads

$$
\mathcal{Z}= \begin{cases}\operatorname{Tr}\left[\left(\mathbf{I}-\mathbf{R}_{2}^{*} \mathbf{R}_{2}\right) \mathbf{D}\left(\mathbf{I}-\mathbf{R}_{1}^{*} \mathbf{R}_{1}\right) \mathbf{D}^{*}\right], & q<\omega / c \\ \operatorname{Tr}\left[\left(\mathbf{R}_{2}^{*}-\mathbf{R}_{2}\right) \mathbf{D}\left(\mathbf{R}_{1}^{*}-\mathbf{R}_{1}\right) \mathbf{D}^{*}\right] e^{-2\left|\beta_{0}\right| d}, & q>\omega / c\end{cases}
$$

where $q$ and $\beta_{0}=\sqrt{(\omega / c)^{2}-q^{2}}$ are the in-plane and out-of-plane wave vectors, respectively. The identity matrix is denoted as $\mathbf{I}$. The reflection coefficient matrix for the interface between vacuum and the FMI $i$ is

$$
\mathbf{R}_{i}=\left[\begin{array}{cc}
r_{s s}^{i} & r_{s p}^{i} \\
r_{p s}^{i} & r_{p p}^{i}
\end{array}\right]
$$

The Fabry-Perot-like matrix reads $\mathbf{D}=\left(\mathbf{I}-\mathbf{R}_{1} \mathbf{R}_{2} e^{2 i \beta_{0} d}\right)^{-1}$.

The heat transfer coefficient, which is defined as $\kappa(T) \equiv \lim _{\Delta T \rightarrow 0} J / \Delta T$, is expressed as

$$
\kappa(T)=\int_{0}^{\infty} \frac{d \omega}{2 \pi} \hbar \omega N^{\prime} \int_{0}^{\infty} \frac{d q}{2 \pi} q \int_{0}^{2 \pi} \frac{d \phi}{2 \pi} Z(\omega, q, \phi),
$$

where the derivative of the Bose-Einstein distribution with respect to the temperature is expressed as

$$
N^{\prime} \equiv \partial N / \partial T=\frac{\hbar \omega e^{\hbar \omega /\left(k_{B} T\right)}}{k_{B} T^{2}\left[e^{\hbar \omega /\left(k_{B} T\right)}-1\right]^{2}} .
$$

For the case of $\phi=0$, the reflection coefficient for the $s$-polarized mode can be expressed as

$$
r_{s s}=\frac{\beta_{0}-\left(\beta_{1} \mu_{r}-i q \mu_{i}\right) /\left(\mu_{r}^{2}-\mu_{i}^{2}\right)}{\beta_{0}+\left(\beta_{1} \mu_{r}-i q \mu_{i}\right) /\left(\mu_{r}^{2}-\mu_{i}^{2}\right)}
$$

and the other reflection coefficients vanish. The photonic transmission coefficient can be expressed as,

$$
\mathcal{Z}(\omega, q, \phi=0)=\frac{4\left[\operatorname{Im}\left(r_{s s}\right)\right]^{2} e^{-2\left|\beta_{0}\right| d}}{\mid 1-r_{s s}^{2} e^{-\left.2\left|\beta_{0}\right| d\right|^{2}}}
$$


from which one can obtain the nonreciprocal dispersion of the symmetric and asymmetric SMP modes between two FMI slabs.

\section{Permeability tensor components of uniaxial FMI}
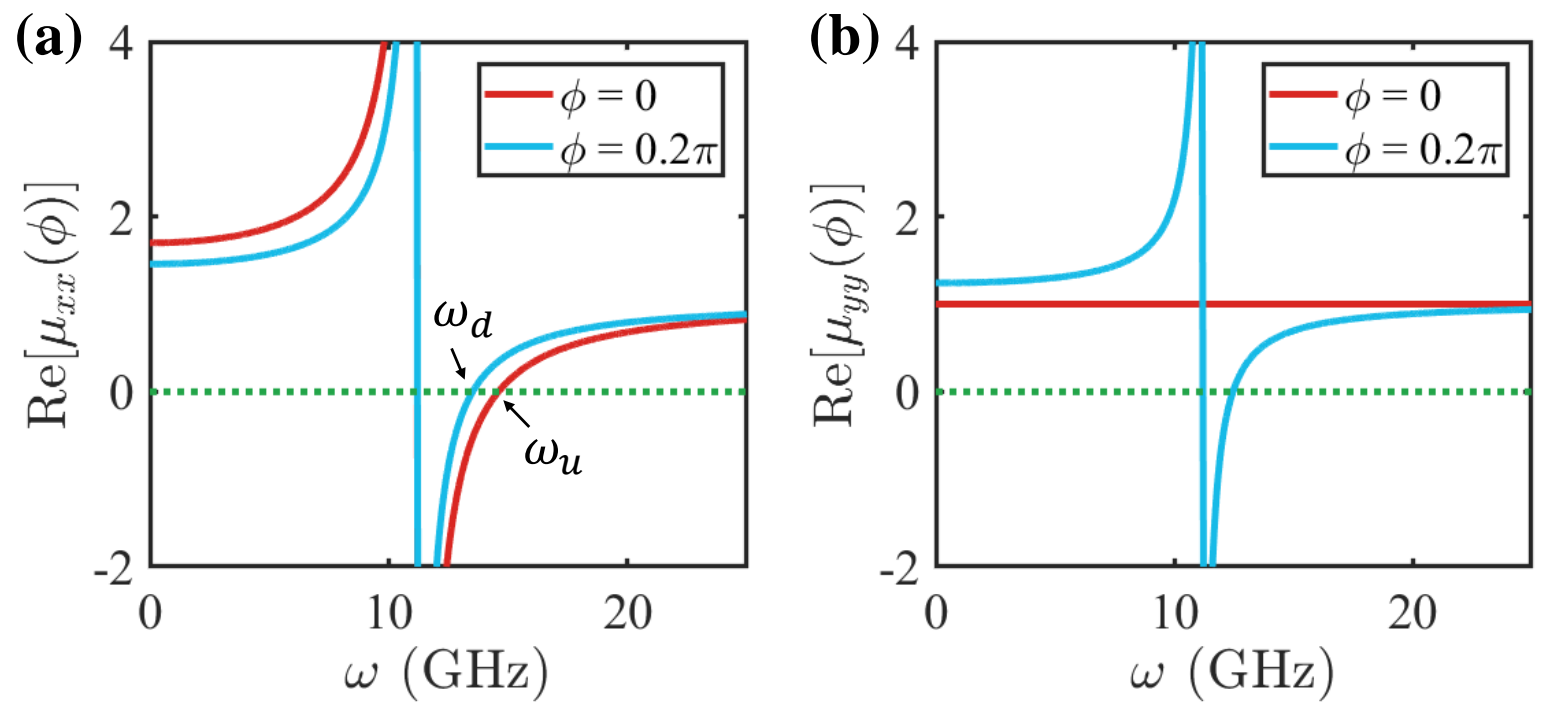

Figure 5: (a-b) The real part of diagonal term of permeability tensor with different azimuthal angle. The damping factor is $\alpha=0.01$.

In our calculation, we use the parameters of YIG and rewrite the permeability tensor as below:

$$
\mu=\left[\begin{array}{ccc}
\mu_{r} & 0 & -i \mu_{i} \\
0 & 1 & 0 \\
i \mu_{i} & 0 & \mu_{r}
\end{array}\right]
$$

After a $\phi$ rotation in $x-y$ plane, the rotated permeability tensor regards as:

$$
\mu(\phi)=\left[\begin{array}{ccc}
\mu_{r} \cos (\phi)^{2}+\sin (\phi)^{2} & \cos (\phi) \sin (\phi)\left(\mu_{r}-1\right) & -i \mu_{i} \cos (\phi) \\
\cos (\phi) \sin (\phi)\left(\mu_{r}-1\right) & \cos (\phi)^{2}+\mu_{r} \sin (\phi)^{2} & -i \mu_{i} \sin (\phi) \\
i \mu_{i} \cos (\phi) & i \mu_{i} \sin (\phi) & \mu_{r}
\end{array}\right]
$$


Fig. 5 shows the real part of $\mu_{x x}$ and $\mu_{y y}$ with different azimuthal angle and $\mu_{z} z=\mu_{r}=\mu_{x x}(\phi)=0$. It indicates value of $\omega_{u}$ and $\omega_{d}$ presented in the main page at the $\mu$-near-zero frequency.

\section{References}

(1) Planck, M.; Masius, M. The Theory of Heat Radiation; P. Blakiston's Son \& Co, New York, 1914.

(2) Cahill, D. G.; Braun, P. V.; Chen, G.; Clarke, D. R.; Fan, S.; Goodson, K. E.; Keblinski, P.; King, W. P.; Mahan, G. D.; Majumdar, A.; Maris, H. J.; Phillpot, S. R.; Pop, E.; Shi, L. Nanoscale thermal transport. II. 2003-2012. Appl. Phys. Rev. 2014, 1, 011305.

(3) Polder, D.; Van Hove, M. Theory of Radiative Heat Transfer between Closely Spaced Bodies. Phys. Rev. B 1971, 4, 3303-3314.

(4) Volokitin, A. I.; Persson, B. N. J. Near-field radiative heat transfer and noncontact friction. Rev. Mod. Phys. 2007, 79, 1291-1329.

(5) Song, B.; Fiorino, A.; Meyhofer, E.; Reddy, P. Near-field radiative thermal transport: From theory to experiment. AIP Adv. 2015, 5, 053503.

(6) Cuevas, J. C.; García-Vidal, F. J. Radiative Heat Transfer. ACS Photonics 2018, 5, 38963915.

(7) Liu, X.; Wang, L.; Zhang, Z. M. Near-Field Thermal Radiation: Recent Progress and Outlook. Nanoscale and Microscale Thermophys. Eng. 2015, 19, 98-126.

(8) Kim, K.; Song, B.; Fernández-Hurtado, V.; Lee, W.; Jeong, W.; Cui, L.; Thompson, D.; Feist, J.; Reid, M. T. H.; García-Vidal, F. J.; Cuevas, J. C.; Meyhofer, E.; Reddy, P. Radiative heat transfer in the extreme near field. Nature 2015, 528, 387. 
(9) Cui, L.; Jeong, W.; Fernández-Hurtado, V.; Feist, J.; García-Vidal, F. J.; Cuevas, J. C.; Meyhofer, E.; Reddy, P. Study of radiative heat transfer in Ångström- and nanometre-sized gaps. Nat. Commun. 2017, 8, 14479.

(10) Wang, J.-S.; Peng, J. Capacitor physics in ultra-near-field heat transfer. Europhys. Lett. 2017, $118,24001$.

(11) Tang, G.; Wang, J.-S. Heat transfer statistics in extreme-near-field radiation. Phys. Rev. B 2018, 98, 125401.

(12) Tang, G.; Yap, H. H.; Ren, J.; Wang, J.-S. Anomalous Near-Field Heat Transfer in CarbonBased Nanostructures with Edge States. Phys. Rev. Applied 2019, 11, 031004.

(13) Volokitin, A. I.; Persson, B. N. J. Resonant photon tunneling enhancement of the radiative heat transfer. Phys. Rev. B 2004, 69, 045417.

(14) Iizuka, H.; Fan, S. Analytical treatment of near-field electromagnetic heat transfer at the nanoscale. Phys. Rev. B 2015, 92, 144307.

(15) Boriskina, S. V.; Tong, J. K.; Huang, Y.; Zhou, J.; Chiloyan, V.; Chen, G. Enhancement and Tunability of Near-Field Radiative Heat Transfer Mediated by Surface Plasmon Polaritons in Thin Plasmonic Films. Photonics 2015, 2, 659-683.

(16) Ilic, O.; Jablan, M.; Joannopoulos, J. D.; Celanovic, I.; Buljan, H.; Soljačić, M. Near-field thermal radiation transfer controlled by plasmons in graphene. Phys. Rev. B 2012, 85, 155422.

(17) Ramirez, F. V.; Shen, S.; McGaughey, A. J. H. Near-field radiative heat transfer in graphene plasmonic nanodisk dimers. Phys. Rev. B 2017, 96, 165427.

(18) Yu, R.; Manjavacas, A.; García de Abajo, F. J. Ultrafast radiative heat transfer. Nat. Commun. 2017, 8, 2 .

(19) Peng, J.; Wang, J.-S. Current-Induced Heat Transfer in Double-Layer Graphene. 
(20) Zhang, Y.; Yi, H.-L.; Tan, H.-P. Near-Field Radiative Heat Transfer between Black Phosphorus Sheets via Anisotropic Surface Plasmon Polaritons. ACS Photonics 2018, 5, 3739-3747.

(21) Rousseau, E.; Laroche, M.; Greffet, J.-J. Radiative heat transfer at nanoscale mediated by surface plasmons for highly doped silicon. Appl. Phys. Lett. 2009, 95, 231913.

(22) Fernández-Hurtado, V.; García-Vidal, F. J.; Fan, S.; Cuevas, J. C. Enhancing Near-Field Radiative Heat Transfer with Si-based Metasurfaces. Phys. Rev. Lett. 2017, 118, 203901.

(23) DeSutter, J.; Tang, L.; Francoeur, M. A near-field radiative heat transfer device. Nat. Nanotechnol. 2019, 14, 751-755.

(24) Ito, K.; Matsui, T.; Iizuka, H. Thermal emission control by evanescent wave coupling between guided mode of resonant grating and surface phonon polariton on silicon carbide plate. Appl. Phys. Lett. 2014, 104, 051127.

(25) Shen, S.; Narayanaswamy, A.; Chen, G. Surface Phonon Polaritons Mediated Energy Transfer between Nanoscale Gaps. Nano Lett. 2009, 9, 2909-2913.

(26) Mulet, J.-P.; Joulain, K.; Carminati, R.; Greffet, J.-J. ENHANCED RADIATIVE HEAT TRANSFER AT NANOMETRIC DISTANCES. Microscale Thermophys. Eng. 2002, 6, 209222.

(27) Iizuka, H.; Fan, S. Analytical treatment of near-field electromagnetic heat transfer at the nanoscale. Phys. Rev. B 2015, 92, 144307.

(28) Chiloyan, V.; Garg, J.; Esfarjani, K.; Chen, G. Transition from near-field thermal radiation to phonon heat conduction at sub-nanometre gaps. Nat. Commun. 2015, 6, 6755.

(29) Song, B.; Ganjeh, Y.; Sadat, S.; Thompson, D.; Fiorino, A.; Fernández-Hurtado, V.; Feist, J.; Garcia-Vidal, F. J.; Cuevas, J. C.; Reddy, P.; Meyhofer, E. Enhancement of near-field radiative heat transfer using polar dielectric thin films. Nat. Nanotechnol. 2015, 10, 253. 
(30) Matsuura, J.; Fukui, M.; Tada, O. ATR mode of surface magnon polaritons on YIG. Solid State Communications 1983, 45, 157 - 160.

(31) Voss, R.; Kotzott, R.; Merten, L. Dispersion of Magnon-Polaritons in Uniaxial Ferrites. physica status solidi (b) 1985, 128, 159-167.

(32) Macêdo, R.; Camley, R. E. Engineering terahertz surface magnon-polaritons in hyperbolic antiferromagnets. Phys. Rev. B 2019, 99, 014437.

(33) Polder, D. On the theory of ferromagnetic resonance. The London, Edinburgh, and Dublin Philosophical Magazine and Journal of Science 1949, 40, 99-115.

(34) Miller, D. A. B.; Zhu, L.; Fan, S. Universal modal radiation laws for all thermal emitters. Proceedings of the National Academy of Sciences 2017, 114, 4336-4341.

(35) Cao, Y.; Fatemi, V.; Fang, S.; Watanabe, K.; Taniguchi, T.; Kaxiras, E.; Jarillo-Herrero, P. Unconventional superconductivity in magic-angle graphene superlattices. Nature 2018, 556, $43-50$.

(36) Cao, Y.; Chowdhury, D.; Rodan-Legrain, D.; Rubies-Bigorda, O.; Watanabe, K.; Taniguchi, T.; Senthil, T.; Jarillo-Herrero, P. Strange Metal in Magic-Angle Graphene with near Planckian Dissipation. Phys. Rev. Lett. 2020, 124, 076801.

(37) Yu, H.; Liu, G. B.; Tang, J.; Xu, X.; Yao, W. Moiré excitons: From programmable quantum emitter arrays to spin-orbit-coupled artificial lattices. Science Advances 2017, 3, e1701696.

(38) Chen, W.; Sun, Z.; Wang, Z.; Gu, L.; Xu, X.; Wu, S.; Gao, C. Direct observation of van der Waals stacking-dependent interlayer magnetism. Science 2019, 366, 983.

(39) Sunku, S. S.; Ni, G. X.; Jiang, B. Y.; Yoo, H.; Sternbach, A.; Mcleod, A. S.; Stauber, T.; Xiong, L.; Taniguchi, T.; Watanabe, K. a. Photonic crystals for nano-light in Moiré graphene superlattices. Science 2018, 362, 1153-1156. 
(40) Hu, G.; Krasnok, A.; Mazor, Y.; Qiu, C. W.; Alù, A. Moiré Hyperbolic Metasurfaces. Nano Letters 2020, 20, 3217-3224.

(41) Gomez-Diaz, J. S.; Tymchenko, M.; Alu, A. Hyperbolic Plasmons and Topological Transitions Over Uniaxial Metasurfaces. Physical Review Letters 2015, 114, 233901.

(42) Hu, G.; Ou, Q.; Si, G.; Wu, Y.; Alù, A. Topological polaritons and photonic magic angles in twisted $\alpha-\mathrm{MoO}_{3}$ bilayers. Nature 2020, 582, 209-213.

(43) Wu, X.; Fu, C.; Zhang, Z. Influence of hBN orientation on the near-field radiative heat transfer between graphene/hBN heterostructures. Journal of Photonics for Energy 2018, 9, 1.

(44) He, M.; Qi, H.; Ren, Y.; Zhao, Y.; Antezza, M. Magnetoplasmon-surface phonon polaritons' coupling effects in radiative heat transfer. Opt. Lett. 2020, 45, 5148-5151.

(45) Wu, X.; Fu, C.; Zhang, Z. Near-Field Radiative Heat Transfer Between Two $\alpha-\mathrm{MoO}_{3}$ Biaxial Crystals. Journal of Heat Transfer 2020, 142, 1-10.

(46) Luo, M.; Zhao, J.; Antezza, M. Near-field radiative heat transfer between twisted nanoparticle gratings. Applied Physics Letters 2020, 117, 053901.

(47) Zhou, C.-L.; Wu, X.-H.; Zhang, Y.; Yi, H.-L.; Mauro, A. Polariton Topological Transition Effects on Radiative Heat Transfer. arXiv:2011.02263 2020,

(48) Pochi,; Yeh, Optics of anisotropic layered media: A new 4 x 4 matrix algebra. Surface Science Letters 1980, 96, 4153.

(49) See Supplemental Material for derivations and additional details.

(50) Yu, W.; Sun, H.; Gao, L. Magnetic control of Goos-Hänchen shifts in a yttrium-iron-garnet film. Sci. Rep. 2017, 7, 45866.

(51) Macêdo, R.; C. Holland, R.; G. Baity, P.; L. Livesey, K.; L. Stamps, R.; P. Weides, M.; A. Bozhko, D. An Electromagnetic Approach to Cavity Spintronics. arXiv:2007.11483 2020, 
(52) Boventer, I.; Pfirrmann, M.; Krause, J.; Schön, Y.; Kläui, M.; Weides, M. Complex temperature dependence of coupling and dissipation of cavity magnon polaritons from millikelvin to room temperature. Phys. Rev. B 2018, 97, 184420.

(53) Zhang, Z. M. Nano/Microscale Heat Transfer 2nd edition; Springer, Cham, 2020.

(54) Latella, I.; Ben-Abdallah, P. Giant Thermal Magnetoresistance in Plasmonic Structures. Phys. Rev. Lett. 2017, 118, 173902.

(55) Iizuka, H.; Fan, S. Significant Enhancement of Near-Field Electromagnetic Heat Transfer in a Multilayer Structure through Multiple Surface-States Coupling. Phys. Rev. Lett. 2018, 120, 063901. 


\section{Graphical TOC Entry}

Through the 'twist angle' between two ferromagnetic insulators, we introduce a large near-field thermal switch and the nonmonotonic twist manipulation for heat flux. 